\title{
Sobrecarga de informação na tomada de decisão: legitimação de escalas e sua relação com a necessidade de cognição do decisor
}

\section{Overloading information in the decision-making: legitimation of scales and its relationship to the need for cognition of the decision}

\author{
Icaro de Oliveira Vieira ${ }^{1}$, Edelvino Razzolini Filho ${ }^{1}$ \\ ${ }^{1}$ Universidade Federal do Paraná, Curitiba, PR, Brasil
}

Autor para correspondência/Mail to: Icaro de Oliveira Vieira, icaroassistentepessoal@gmail.com

Financiamento/Funding: Fundação Araucária e Governo do Estado do Paraná

Copyright (C) 2019 Vieira \& Razzolini Filhoo. Todo o conteúdo da Revista (incluindo-se instruções, política editorial e modelos) está sob uma licença Creative Commons Atribuição-NãoComercial-Compartilhalgual 3.0 Não Adaptada. Ao serem publicados por esta Revista, os artigos são de livre uso em ambientes educacionais, de pesquisa e não comerciais, com atribuição de autoria obrigatória. Mais informações em http://revistas.ufpr.br/atoz/about/submissions\#copyrightNotice.

\begin{abstract}
Resumo
O desafio que se vislumbra para o processo decisório é a percepção da quantidade de informação como uma variável a ser controlada. A tomada de decisão do gestor, por sua vez, se vincula a sua base cognitiva. O presente trabalho tem como objetivo a busca de evidências de validade psicométrica, através das técnicas critério e construto, de dois instrumentos psicométricos para a mensuração da sobrecarga de informação na tomada de decisão e eventuais relações com a necessidade de cognição do decisor. Para a análise, se pretende apreciar uma amostra formada por estudantes de pós-graduação. Para a legitimação dos instrumentos se utilizará a técnica estatística denominada análise fatorial por ela possuir a capacidade de determinar o grau de influência de uma dada variável na explicação de um fator. Para o tratamento dos dados, se utilizará de diversos métodos estatísticos. Este estudo busca encontrar evidências acerca do impacto que a variável cognição do gestor experimenta na presença de sobrecarga de informação. Suas principais contribuições seriam: (i) a verificação da existência de sobrecarga de informação na tomada de decisão e eventual relação com a necessidade de cognição do decisor; (ii) a mensuração da sobrecarga de informação e a necessidade de cognição do decisor em situações caracterizadas no âmbito organizacional; (iii) elaboração de um quadro conceitual por meio do qual seja possível a visualização de eventual relação entre o volume de informação e a necessidade de cognição do tomador de decisão.
\end{abstract}

Palavras-chave: Parâmetros psicométricos; Análise do comportamento; Legitimação de escalas.

\begin{abstract}
The challenge for the decision-making process is the perception of the amount of information as a variable to be controlled. Management decision-making, in turn, is linked to its cognitive basis. The objective of this study is to search for evidence of psychometric validity, through the criterion and construct techniques, of two psychometric instruments for the measurement of information overload in decision making and possible relationships with the decision maker's need for cognition. For the analysis, if you want to appreciate a sample formed by postgraduate students. For the legitimacy of the instruments will be used the statistical technique called factorial analysis because it has the ability to determine the degree of influence of a given variable in the explanation of a factor. For the treatment of the data, several statistical methods will be used. This study seeks to find evidence about the impact that the manager's cognition variable experiences in the presence of information overload. Its main contributions would be: (i) the verification of the existence of information overload in the decision making and eventual relation with the decision maker's cognitive need; (ii) the measurement of information overload and the decision maker's need for cognition in organizational situations; (iii) elaboration of a conceptual framework through which it is possible to visualize the possible relationship between the volume of information and the cognitive need of the decision maker.
\end{abstract}

Keywords: Psychometric parameters; Behavior analysis; Legitimation of scales.

\section{INTRODUÇÃO}

Os cabeçalhos das seções/subdivisões devem ser breves, claros e não numerados. O texto do artigo deve ser estruturado preferencialmente contemplando os seguintes itens: introdução, método, resultados e considerações finais. Acrônimos e abreviações devem estar entre parênteses e serem precedidos de seu significado completo quando do primeiro uso no texto.

A gestão de uma empresa exige informações relevantes para o processo decisório, conforme Anthony e Govindarajan (2008). Entretanto, a partir da Era da Informação, com a disseminação da internet, têm-se observado os impactos da sobrecarga de informação no comportamento do indivíduo, no nível social e nos processos de tomada de decisão Gleick (2013); Santos (2011); Wurman (2005), que é o principal interesse deste estudo.

A expressão sobrecarga de informação é atribuída a Alvin Toffler, nos idos da década de 70. Para ele, super estimulação cognitiva interfere em nossa capacidade de pensar (Toffler, 1971). Nesse sentido, Castells (1999) afirma que no seio da sociedade opera uma revolução tecnológica concentrada nas tecnologias da informação que está remodelando sua base em ritmo acelerado. Ideia reforçada pela Lei de Moore que afirma que o processamento dos computadores tem sua capacidade dobrada a cada dezoito meses. 
Desta forma, para compensar situações de mudança rápida e irregular ou um contexto carregado de novidade, o indivíduo tem de processar muito mais informações do que antes para tomar decisões efetivas e racionais (Toffler, 1971).

Atribui-se a Simon (1963), segundo Moritz (2006), o titulo de "pai" do Processo Decisório, cujos estudos propõem um modelo de decisão que admite a existência de uma racionalidade limitada. "O indivíduo coloca-se numa situação onde certos estímulos e certas classes de informações terão influência sobre ele" (Simon, 1970, p. 82).

Nesse sentido, o Modelo de Racionalidade Limitada (Bounded Rationality) adverte não ser possível para um tomador de decisão ter acesso a todas as possibilidades de ação devido à impossibilidade física de ter acesso a todas as informações e processá-las, além do alto custo envolvido nesse processo (Moritz, 2006).

Segundo Hambrick and Mason (1984) a tomada de decisão do gestor vincula-se a sua base cognitiva, que é explicada pelos: i) conhecimentos ou suposições sobre fatos futuros; ii) conhecimentos de alternativas e iii) conhecimento dos efeitos atribuídos às alternativas. Além disso, Altoé (2017) afirma que a tomada de decisão reflete os valores do gestor.

Em vista do exposto, a inquietação que se observa é a de se estudar as questões relacionadas à quantidade de informação e a necessidade de cognição do decisor para a tomada de decisão. Portanto, se busca compreender como a sobrecarga de informação afeta a capacidade cognitiva individual em decisões no nível gerencial.

\section{Objetivos da Pesquisa}

Em consonância com a questão de pesquisa estabelecida, os objetivos orientados do presente estudo são:

\section{Objetivo Geral}

O presente trabalho tem como objetivo a busca de evidências de validade psicométrica, através das técnicas critério e construto, de dois instrumentos psicométricos para a mensuração da sobrecarga de informação na tomada de decisão e eventuais relações com a necessidade de cognição do decisor.

\section{Objetivos Específicos}

a) examinar as propriedades psicométricas dos instrumentos utilizados para medir sobrecarga de informação e necessidade de cognição no contexto brasileiro;

b) investigar eventuais relações entre sobrecarga de informação e necessidade de cognição na tomada de decisão;

c) realizar a construção de um quadro conceitual que possibilite a visualização das variáveis e suas eventuais relações.

\section{Principais Contribuições Científicas, Tecnológicas ou de Inovação}

O desafio que se vislumbra para o processo decisório é a percepção da quantidade de informação como uma variável a ser controlada. No estudo de Roberts and O'Reilly (1974) - "Measuring Organizational Communication" - foi desenvolvida uma escala para medição de sobrecarga de informação em um contexto de comunicação intraorganizacional. Jacoby, Speller and Kohn (1974) ao tratarem de escala que mede a sobrecarga definem-na como uma evocação das tentativas dos trabalhadores para processar uma quantidade de informação que excede a sua capacidade para assimilá-las ou processá-las durante um período. Essas manifestações da sobrecarga de informação estão aumentando erros na execução do trabalho e criando confusão e frustração nos funcionários (Jacoby, Speller \& Kohn, 1974; Keller \& Staelin, 1987).

Esta proposta amplia e potencializa o estudo anteriormente proposto uma vez que, por meio dela, se legitimará dois instrumentos psicométricos para a mensuração de sobrecarga de informação na tomada de decisão, seguindo critérios de verificação de estudos de validade (Cronbach, 1996; Noronha \& Alchieri, 2002). O método é essencialmente estatístico, pois está baseado em coleta, tratamento e análise de dados.

A validade é um dos mais relevantes aspectos considerados para a construção de testes psicológicos. Segundo Anastasi e Urbina (2000, p. 107), "refere-se àquilo que um teste mede e a quão bem ele faz isso". Fachel e Camey (2000) atestam que um teste é válido quando ele mede o que o pesquisador deseja e pensa que está medindo.

As verificações das evidências de validade que esse estudo realizará serão as validações por meio de critério e construto. Para Pasquali (2001) a validade de critério refere-se ao grau de eficácia que o teste apresenta em predizer determinado desempenho de um sujeito.

Já a validade de construto, segundo Van Kolck (1981), tem por objetivo pesquisar as qualidades psicológicas que o teste mede e pode ser definida como o "grau pelo qual o teste mede um construto teórico ou traço para o qual 
ele foi designado" (Fachel \& Camey, 2000, p. 164). Para Pasquali (2001) esse tipo de validade é a forma mais fundamental de validade.

Como contribuição científica destaca-se verificar, complementar e propor alterações no modelo inicialmente proposto por Roberts and O'Reilly (1974), no contexto brasileiro e no âmbito organizacional, por meio da investigação empírica. O delineamento metodológico, bem como os procedimentos e técnicas de coleta e análise dos dados proporcionará a crítica de conceitos de parâmetros inicialmente utilizados.

As principais contribuições seriam: (i) a verificação da existência de sobrecarga de informação na tomada de decisão e eventual relação com a necessidade de cognição do decisor; (ii) a mensuração da sobrecarga de informação e a necessidade de cognição do decisor em situações caracterizadas no âmbito organizacional; (iii) elaboração de um quadro conceitual por meio do qual seja possível a visualização de eventual relação entre o volume de informação e a necessidade de cognição do tomador de decisão.

Outro fator considerado importante é o enfoque multidisciplinar da proposta. A elaboração desta proposta será executada com estudos de várias áreas, por envolver a utilização de informações multidisciplinares. Vieira e Razzolini (2018) relatam que a maior quantidade de publicações sobre sobrecarga de informação na tomada de decisão está na área de Sistemas de Suporte à Decisão, em segundo lugar na Psicologia, seguida das áreas de Contabilidade, Tecnologia da Informação e Administração.

Como potenciais impactos científicos destaca-se: (i) divulgação de forma ampla a fim de possibilitar o aumento do criticismo sobre o tema sobrecarga de informação e tomada de decisão (ii) revisão de conceitos estabelecidos; (iii) análise de relações anteriormente esboçadas; (iv) fortalecimento de associação entre grupos e pesquisadores nacionais e internacionais que desenvolvem o tema.

Como potenciais impactos tecnológicos destaca-se o desenvolvimento de quadro conceitual com a relação entre o volume de informação e necessidade de cognição na tomada de decisão, com aplicação de tecnologias sócio organizacionais. Como potenciais impactos econômicos destaca-se a aplicação de resultados do quadro conceitual para auxiliar a decisão de investimento e fomento de organismos econômicos e sociais tanto na iniciativa pública quanto na privada.

Como potenciais impactos sociais e culturais, só com o desenho de pesquisa aqui apresentado é possível perceber as respostas dos tomadores de decisão quanto à funcionalidade do quadro conceitual que será o produto final deste trabalho. O foco da proposta deve estar permanentemente de acordo com as premissas da tecnologia sócio organizacional e do desenvolvimento tecnológico organizacional.

\section{REVISÃO DE LITERATURA}

Jacoby, Speller and Kohn (1974) apresentaram um primeiro passo para lidar com as complexidades do comportamento de decisão na presença de carga de informação. Eles concluíram que os consumidores sentem-se mais satisfeitos e menos confusos com pouca carga de informação enquanto que tomam decisões de compra mais pobres na presença de mais informações.

Vieira e Razzolini (no prelo) ao gerarem um portfólio sobre o tema sobrecarga de informação e tomada de decisão, elencaram os artigos mais relevantes no período entre 2007 e 2016.

- Kuksov and Villas-Boas (2010) demonstram que quando as alternativas aos consumidores abrangem a preferência, a avaliação pode leva-los a não procurar e não escolher se muitas ou poucas alternativas são oferecidas.

- A motivação de pesquisa de Chen, Shang and Kao (2009) foi a reavaliação da concepção da qualidade da decisão, do estado subjetivo para a decisão e limiar de carga de informação.

- Cäniels and Bakens (2012) estudam o efeito da tomada de decisão em um ambiente de projeto múltiplo. Utilizando modelagem de equações estruturais concluíram que o uso de um sistema de informação é vantajoso para gerentes de projeto.

- A experiência empírica promovida por Cheung, Xiao and Liu (2014) investiga como indicações de informação social em comunidades sociais on-line podem influenciar as decisões de compra do consumidor.

- Gao, Zhang, Wang and Ba (2012) examina os diferentes papéis da quantidade e qualidade da informação e sua relação com a decisão dos consumidores na presença de muitas informações.

A sobrecarga de informação na tomada de decisão é apenas mencionada por Avelino, Löbler e Flaviano (2015) uma vez que o objetivo de seu estudo é verificar a influência da motivação epistêmica no processo decisório. O estudo conclui que não há diferenças significativas entre as distribuições das escolhas feitas com base nas diferentes condições motivacionais. 
Considerando o número reduzido de estudos sobre sobrecarga de informação e tomada de decisão e por não haver sido encontrada qualquer pesquisa a respeito no contexto brasileiro, pretende-se, com a presente pesquisa, empreender esforços para suprir essa lacuna.

\section{PROCEDIMENTOS METODOLÓGICOS}

Para responder à questão de pesquisa, propõe-se uma hipótese com a finalidade de conduzir a verificação empírica dos objetivos definidos que se alinham ao problema a ser pesquisado. A hipótese, denominada H1, sustenta que, quanto maior for o nível de cognição dos respondentes, maior será a presença de sobrecarga de informação.

Na coleta de dados, a proposta foi concebida com, além das perguntas com os proxies das características observáveis dos decisores (Altoé, 2017) (idade, nível de formação, experiência profissional, tempo no cargo e gênero), disponibilizadas no final, os participantes responderão os seguintes instrumentos:

Adaptação de escala para medição de sobrecarga de informação utilizada no estudo de Roberts and O’ Reilly (1974) - "Measuring Organizational Communication".

Escala Need For Cognition (NFC). Elaborada originalmente por Cacioppo, Petty, and Feng Kao (1984), reúne 18 itens que visam avaliar a tendência de um indivíduo em se engajar em atividades que exigem esforço analítico ou gostar de pensar (por exemplo, Prefiro problemas complexos aos simples) que são respondidos em escala de sete pontos $(1=$ Discordo totalmente a $7=$ concordo totalmente). Evidências acerca de sua estrutura fatorial foram descritas, sugerindo-se um fator geral dominante (Cacioppo \& Petty, 1982). Contudo, encontram-se estudos em que se observam diferentes soluções fatoriais, tais como com três fatores (Tanaka, Panter, \& Winborne, 1988) ou com cinco (Lord \& Putrevu, 2006). A consistência interna (alfa de Cronbach) do fator geral tem sido aceitável (a > 0,70) (Nunnaly, 1991; Pasquali, 2003). Deve-se ressaltar ainda, a estabilidade temporal desse construto, compreendido como uma característica da personalidade, ao descrever pessoas que tendem a se envolver em atividades cognitivas, obtendo, assim, satisfação com sua realização (Cacioppo, Petty, Feistein \& Jarvis, 1996; Silva \& Garcia-Marques, 2006). O estudo de Carnevale, Inbar and Lerner (2011) demonstra que a NFC está correlacionada com melhor desempenho em tomada de decisões.

Pretende-se analisar uma amostra não-probabilística formada por estudantes de pós-graduação de Curitiba (PR). A amostra será composta por alunos da IES Universidade Federal do Paraná, escolhidos entre diferentes cursos de doutorado, mestrado e especialização do setor de Ciências Sociais Aplicadas da referida IES.

Tendo em vista que um dos objetivos do estudo é comparar resultados de profissionais com alto e baixo envolvimento com decisões gerenciais, escolher-se-á, de forma proposital, estudantes das áreas de Administração, Finanças, e Contabilidade, pois, segundo Lima, Bruni e Menezes (2013), essa seria a característica mais frequente em profissionais dessas áreas.

Por sua vez, Beltramini (1988); Toffler (1971) asseveram que pode haver uma relação entre complexidade, manipulação de grandes números e requisitos crescentes de informação. Esses autores sugerem que profissionais que estão submetidos a mudanças frequentes de tecnologia em seu trabalho podem apresentar sobrecarga de informação.

Pretende-se que o uso de amostra diversificada permita segregar os respondentes em função de seu variado nível de envolvimento com decisões gerenciais e exposição à sobrecarga de informação e agrupá-los em dois níveis distintos. O tamanho da amostra seguirá a orientação de Hair, Anderson, Tatham and Black (1998); com não menos de trinta sujeitos por célula de pesquisa e com as células apresentando quantidade próxima de observações.

Liyanarachchi and Milne (2005), Elliott, Hodge, Kennedy and Pronk (2007), Lord and Putrevu (2006), Silva e Garcia-Marques (2006) sugerem em seus trabalhos que estudantes, seja de graduação ou de pós-graduação, podem ser utilizados em pesquisas acadêmicas, pois representam bons substitutos para as pesquisas realizadas com profissionais.

Na análise de dados, com o intuito de atingir o primeiro objetivo específico (examinar as propriedades psicométricas dos instrumentos), pretende-se calcular as estatísticas descritivas (por exemplo, média, desvio padrão e frequência) e a consistência interna do instrumento (alfa de Cronbach) (Clark \& Watson, 1995; Pasquali, 2010). Em seguida, pretende-se utilizar a técnica multivariada denominada análise fatorial para o cálculo da matriz de covariâncias que, segundo Lima, Bruni e Menezes (2013); Appel, Gnambs and Maio (2012); Maio and Esses (2001); Byrne (2016) e Tabachnick and Fidell (2013), possui capacidade de determinar o grau de influência de determinada variável na explicação de um fator, além de analisar a intensidade das relações entre as variáveis observadas e, a partir delas, estimar um modelo fatorial subjacente capaz de reproduzir essas relações (objetivos 2 e 3 dessa proposta). Segundo Byrne (2016) e Tabachnick and Fidell (2013) o qui-quadrado (X2) testa a probabilidade do modelo teórico se ajustar aos dados já que ele verifica a dependência ou independência entre as variáveis.

Pretende-se também utilizar o Teste de Esfericidade de Bartlett com o intuito de confirmar a validade da aplicação da análise fatorial ao conjunto de variáveis desse estudo uma vez que, segundo Hair, Anderson, Tatham 
and Black (1998), ele é empregado para testar hipótese nula em que os itens na matriz de correlação não estão correlacionados, o que, caso aconteça, atestará que a análise fatorial da escala é apropriada.

Por fim, com o intuito de atingir o segundo objetivo (investigar eventuais relações sobre sobrecarga de informação e necessidade de cognição), pretende-se utilizar o teste estatístico ANOVA para o cálculo da variância dento dos grupos e entre os grupos e a correlação r de Pearson com vistas a conhecer a associação de NFC à sobrecarga de informação.

\section{CRONOGRAMA DA PESQUISA}

Para realizar esta pesquisa, as seguintes atividades, expostas no quadro a seguir, serão necessárias.

Cronograma de ações de pesquisa:

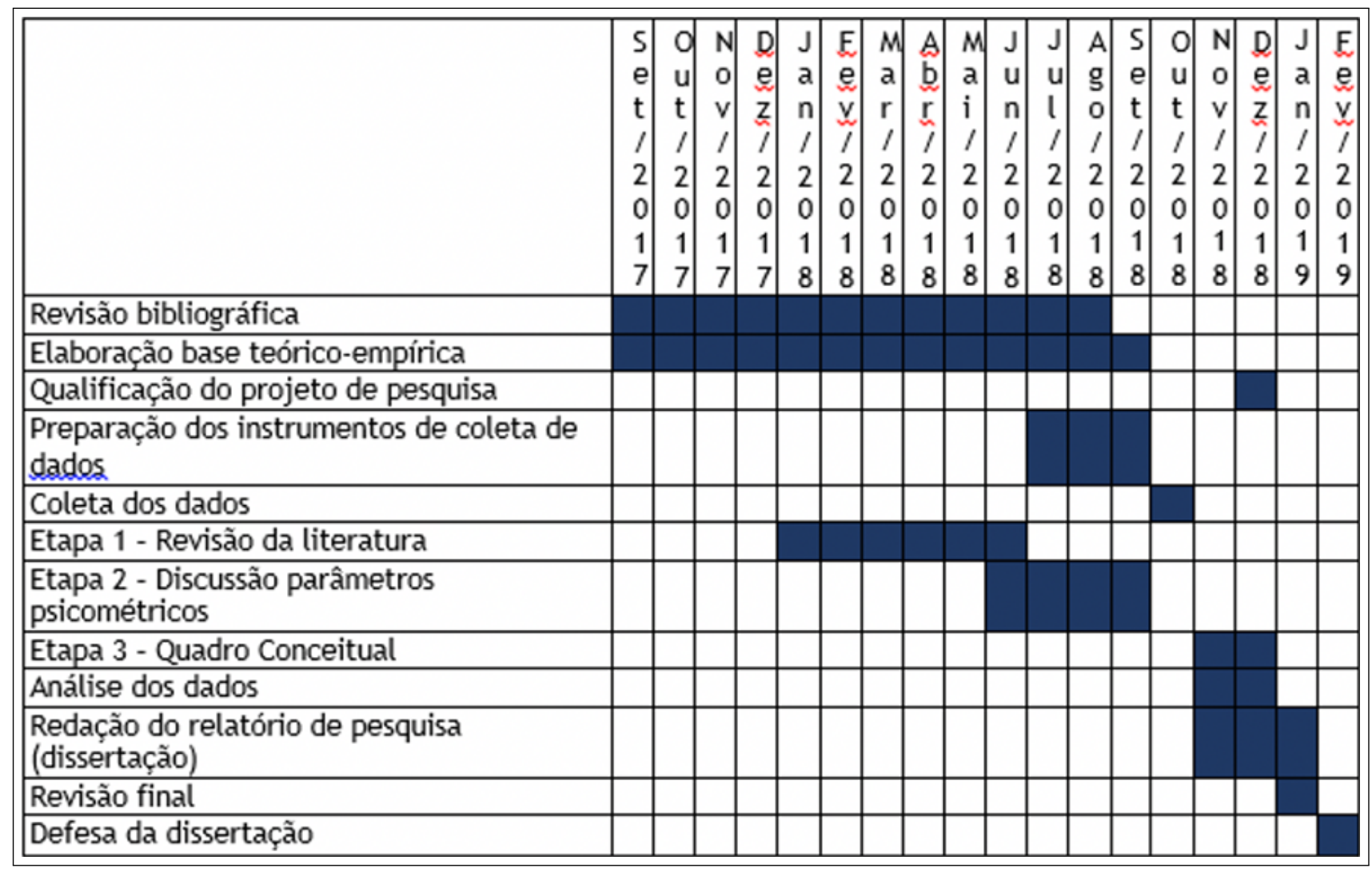

Figura 1. Cronograma de pesquisa

Fonte: Elaborado pelos autores (2019)

\section{CONSIDERAÇÕES PARCIAIS}

Este estudo busca encontrar evidências acerca do impacto que a variável sobrecarga de informação experimenta a partir da necessidade de cognição do tomador de decisão. Espera-se confirmar a H1 partindo do pressuposto de que o indivíduo que apresenta um nível elevado de cognição possa tender a buscar, por si só, um nível elevado de carga informacional.

\section{AGRADECIMENTOS}

FUNDACÃO ARAUCARII

Apoio ao Desenvolvimento Cientifico

e Tecnológico do Paraná
PARANÁ GOVERNO DO ESTADO
À Fundação Araucária e ao Governo do Estado pelo apoio financeiro para a realização do evento. 


\section{REFERÊNCIAS}

Altoé, S. M. L. (2017). Associação dos estilos de liderança no uso do sistema de controle gerencial e o efeito moderador das características observáveis de gestores de topo: evidências em empresas familiares do segmento supermercadista. Tese de doutorado, Universidade Federal do Paraná, Paraná.

Anastasi, A., \& Urbina, S. (2000). Testagem psicológica. Porto Alegre: Artmed.

Anthony, R. N., \& Govindarajan, V. (2008). Sistemas de controle gerencial (12a ed.). São Paulo: McGraw-Hill.

Appel, M., Gnambs, T., \& Maio, G. R. (2012). A short measure of the need for affect. Journal of Personality Assessment, 94(4), p. 418-426.

Avelino, A. F. A.; Löbler, M. L. \& Flaviano, V. (2015). Sobrecarga de informação no processo decisório: O papel da motivação epistêmica na resposta individual. Anais do Encontro Nacional da Associação Nacional de Pós-Graduação e Pesquisa em Administração (Enanpad), Belo Horizonte, MG, Brasil, 39.

Beltramini, R. F. (1988). High technology salespeople's information acquisition strategies. Journal of Personal Selling 83 Sales Management, 8(1), p. 37-44.

Byrne, B. M. (2016). Structural equation modeling with AMOS: basic concepts, applications, and programming. Londres: Routledge.

Cacioppo, J. T., \& Petty, R. E. (1982). The need for cognition. Journal of Personality and Social Psychology, v. 42(1), p. 116.

Cacioppo, J. T., Petty, R. E., \& Feng Kao, C. (1984). The efficient assessment of need for cognition. Journal of Personality Assessment, 48(3), p. 306-307.

Cacioppo, J. T., Petty, R. E., Feistein, J. A., \& Jarvis, B. G. (1996). Dispositional differences in cognitive motivation: the life and times of individuals varying in need for cognition. Psychological Bulletin, 119(2), p. 197-253.

Cäniels, M. C. J., \& Bakens, R. J. J. M. (2012). The effects of project management information systems on decision making in a multi project environment. International Journal of Project Management, 30(2), p. $162-175$.

Carnevale, J. J., Inbar, Y., \& Loerner, J. S. (2011). Individual differences in need for cognition and decisionmaking competence among leaders. Personality and Individual Differences, 51(3), p. 274-278.

Castells, M. (1999). Sociedade em rede. São Paulo: Paz e Terra.

Chen, Y., Shang, R., \& Kao, C. (2009). The effects of information overload on consumers' subjective state towards buying decision in the internet shopping environment. Eletronic Commerce Research and Applications, $8(1)$, p. $48-58$.

Cheung, C. M. K., Xiao, B. S., \& Liu, I. L.B. (2014). Do actions speak louder than voices? The signaling role of social information cues in influencing consumer purchase decisions. Decision Support Systems, 65, p. $50-58$.

Clark, L. A., \& Watson, D. (1995). Constructing validity: basic issues in objective scale development. Psychological Assessment, 7(3), p. 309.

Cronbach, L. (1996). Fundamentos da testagem psicológica. Porto Alegre: Artmed.

Elliott, W. B., Hodge, F., Kennedy, J., \& Pronk, M. (2007). Are MBA students a good proxy for nonprofessional Investors? The Accounting Review, 82(1), p. 139- 168.

Fachel, J. M. G., \& Camey, S. (2000). Avaliação psicométrica: a qualidade das medidas e o entendimento dos dados. Psicodiagnóstico, 5, p. 158-170.

Gao, J., Zhang, C., Wang, K., \& Ba, S. (2012). Understanding online purchase decision making: the effects of unconscious thought, information quality, and information quantity. Decision Support Systems, 53(4), p. $772-781$.

Gleick, J. (2013). A informação: uma história, uma teoria, uma enxurrada. São Paulo: Companhia das Letras.

Hair, J. F., Anderson, R., Tatham, R., \& Black, W. (1998). Multivariate data analysis. Upper Saddie River, NJ: Prentice Hall.

Hambrick, D. C., \& Mason, P. A. (1984). Upper echelons: the organization as a reflection of its top managers. Academy of Management Review, 9(2), p. 193 - 206. 
Jacoby, J., Speller, D. E., \& Kohn, C. A. (1974). Brand choice behavior as a function of information load. Journal of Marketing Research, 11(1), p. 63-69.

Keller, K. L., \& Staelin, Richard. (1987). Effects of quality and quantity of information on decision effectiveness. Journal of consumer research, 14(2), p. 200-213.

Kuksov, D., \& Villas-Boas, J. M. (2010). When more alternatives lead to less choice. Marketing Science, 29(3), p. $507-524$.

Lima, R. N., Filho, Bruni, A. L., \& Menezes, I. G. (2013). Validação do teste need for cognition: um estudo em contabilidade comportamental. Psicologia: Ciência e Profissão, 33(1), p. 112-131.

Liyanarachchi, G. A., \& Milne, M. J. (2005). Comparing the investment decisions of accounting practitioners and students: an empirical study on the adequacy of student surrogates. Accounting Forum, 29(2), 121-135. Recuperado de 10.1016/j.accfor.2004.05.001

Lord, K. R., \& Putrevu, S. (2006). Exploring the dimensionality of the need for cognition scale. Psychology \&6 Marketing, 23(1), p. 11-34.

Maio, G. R., \& Esses, V. M. (2001).The need for affect: individual differences in the motivation to approach or avoid emotions. Journal of Personality, 69(4), p. 583- 614.

Moritz, G. O. (2006). Processo decisório. Florianópolis: SEAD/UFSC.

Noronha, A. P. P., \& Alchieri, J. C. (2002). Reflexões sobre os instrumentos de avaliação psicológica. In PRIMI, Ricardo. (Org.). Temas em Avaliação Psicológica. Campinas: IBAP, p. 7-16.

Nunnally, J. C. (1991). Teoría psicométrica. México: Trillas.

Pasquali, L. (2010). Instrumentação psicológica: fundamentos e Práticas. Porto Alegre: Artmed.

Pasquali, L. (2001). Técnicas de exame psicológico - TEP: manual (v. 1). São Paulo: Casa do Psicólogo.

Pasquali, L. (2003). Psicometria: teoria dos testes na psicologia e na educação. Petrópolis, RJ: Vozes.

Roberts, K. H., \& O'Reilly, C. A. (1974). Measuring organizational communication. Journal of Applied Psychology, 59(3), p. 321.

Santos, L. G. (2011). Politizar as novas tecnologias: o impacto sociotécnico da informação digital e genética. São Paulo: Editora 34.

Silva, J., \& Garcia-Marques, T. (2006). Medindo necessidade de cognição e fé na intuição: tradução e adaptação das duas escalas à população Portuguesa. Laboratório Psicologia, 4(1), p. 29-43.

Simon, H. A. (1963). A capacidade de decisão e liderança. Rio de Janeiro: Fundo de Cultura.

Simon, H. A. (1970). Comportamento administrativo: estudo dos processos decisórios nas organizações (2a ed.). Rio de Janeiro: Fundação Getúlio Vargas.

Tabachnick, B. G., \& Fidell, L. S. (2013). Using multivariate statistics. Needham Heights, MA, USA: Allyn \& Bacon/Pearson Education.

Tanaka, J. S., Panter, A. T., \& Winborne, W. C. (1988). Dimensions of the need for cognition: subscales and genderd differences. Multivariate Behavioral Research, 23(1), p. 35-50.

Toffler, A. (1971). Future shock. New York: Bantam.

Van Kolck, O. L. (1981). Técnicas de exame psicológico e suas aplicações no Brasil. Petrópolis: Vozes.

Vieira, I. O., \& Razzolini, E., Filho. (2018). Estado do conhecimento sobre sobrecarga de informação na tomada de decisão em artigos científicos no período ente 2007 e 2016. Anais do Encontro de Estudos Sobre Empreendedorismo e Gestão de Pequenas Empresas (Egepe), São Paulo, SP, Brasil, 10.

Vieira, I. O., \& Razzolini, E., Filho. (no prelo). Sobrecarga de informação e processo decisório: uma discussão por meio de revisão sistemática. Anais do Encontro Nacional da Associação Nacional de Pós-Graduação e Pesquisa em Administração (Enanpad), Curitiba, PR.

Wurman, R. S. (2005). Ansiedade de informação 2. São Paulo: Editora Cultura. 